Rev. salud pública. 10 (5):744-755, 2008

\title{
Validación de Índices Pronósticos de Infección del Sitio Quirúrgico en Hospitales de Colombia
}

\author{
Validating prognostic surgical site infection indices from \\ hospitals in Colombia
}

Nancy Yomayusa ${ }^{1}$, Hernando Gaitán ${ }^{1}$, Isabel Suárez ${ }^{1}$, Milciades Ibáñez ${ }^{1}$, Paula Hernandez ${ }^{1}$, Carlos Álvarez ${ }^{1}$, Mónica Sossa ${ }^{1}$, Hernando Altahona ${ }^{1}$, Álvaro Arango ${ }^{2}$ y Otto Susman ${ }^{3}$

\begin{abstract}
1 Departamento de Clínicas Médicas, Departamento de Epidemiología, Departamento de Enfermedades Infecciosas, Instituto de Investigaciones. Clínica Colsanitas S.A - Fundación Universitaria Sanitas. Bogotá, Colombia._nyomayusa@colsanitas.com, hggaitand@cable.net.co, icsuarez@colsanitas.com, mippalad@yahoo.com,calvarez@colsanitas.com,mocasossa1@yahoo.com, haltahona@unisanitas.edu.co, phrueda@yahoo.es

2 Fundación Cardio Infantil.Bogotá,Colombia. aarango@cardioinfantil.org

3 Departamento de Infectología. Fundación Abood A Shaio. Bogotá, Colombia. osussmann@gmail.com
\end{abstract}

Recibido 25 Marzo 2008/Enviado para Modificación 5 Octubre 2008/Aceptado 13 Noviembre 2008

\section{RESUMEN}

Objetivo Establecer la capacidad predictiva para infección del sitio quirúrgico (ISQ) de los índices de riesgo del National Nosocomial Infections Surveillance System (NNIS) y Study on the Efficacy of Nosocomial Infection Control (SENIC) en cinco hospitales y, evaluar la capacidad predictiva de otros factores de riesgo.

Métodos Cohorte prospectiva de pacientes sometidos a cirugía entre julio de 2006 a febrero de 2007 en cinco hospitales de Colombia. Se definió ISQ según los criterios del CDC. Se evaluaron variables como: edad, género, coomorbilidad, tipo de cirugía, herida, especialidad, tiempo quirúrgico y desenlace. Se evaluó el desempeño operativo de los índices usando el área bajo la curva operador receptor; se construyó un modelo predictivo usando un modelo de regresión logística incondicional con las variables asociadas a infección en el análisis bivariado y/o aquellas conocidas por estudios previos.

Resultados Fueron evaluados 7022 procedimientos quirúrgicos con una tasa de ISQ de 2,9\%. El rendimiento de los índices de riesgo NNIS y SENIC fue muy similar para predecir ISQ (área bajo la curva de 0,682 IC95 \% 0,641-0,710 y 0,668 IC95 \% $0.641-0.722$, respectivamente). Se construyó un modelo predictivo que incluía variables del NNIS y SENIC, además de edad, antecedente de diabetes, transfusiones y especialidad quirúrgica el cual mostró un desempeño de 0,746 (IC95 \% 0,709-0,783), en ISQ superficial de 0,70 (IC95 \% 0,659-0,741), en ISQ profunda de 0,712 (IC95 \% 0,673-0,751) y en ISQ órgano espacio de 0,719 (IC95 \% 0,683-0,755).

Conclusiones Los modelos de predicción existentes para ISQ tienen una moderada capacidad discriminativa pero pueden ser mejorados con algunos factores locales. 
Palabras Clave: Infección de herida operatoria, Colombia, índice de riesgo (fuente: DeCS, BIREME).

\section{ABSTRACT}

Objective Establishing the effectiveness of the National Nosocomial Infection Surveillance System (NNIS) and Study of the Efficacy of Nosocomial Infection Control's (SENIC) prognostic surgical site infection (SSI) indices in Colombian hospitals and assessing the influence of other risk factors.

Methods A prospective, multicentre cohort study was conducted in five Colombian hospitals. All patients undergoing surgery requiring hospitalisation or ambulatory surgeries having a greater risk of infection were enrolled. A case was defined as being those subjects who presented the $C D C$ diagnostic criteria of incisional superficial, deep incisional or organ-space SSI. Age, gender, co-morbidities, type of surgery, procedures, medical specialty, type of wound, surgical time, antibiotic prophylaxis and patient outcome were used for developing a predictive model of SSI using logistical regression analysis. The indexes' predictive ability was assessed by using the area under the receiver operating curve (ROC).

Results 7,022 surgical procedures were evaluated and SSI rate was $2.9 \%$. NNIS and SENIC risk index performance was similar to that for predicting SSI (0.68 cf 0.66 area under ROC, respectively). The new predictive model involved other factors such as age, diabetes mellitus, transfusions and surgical specialty showing 0.74 operating performance.

Conclusions Existing SSI predictive models have a moderate ability for predicting SSI but this can be improved with some local factors.

Key Words: Surgical wound infection, Colombia, risk index (source: MeSH, NLM).

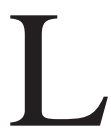
a infección del sitio quirúrgico (ISQ) se constituye en la tercera causa de infección intrahospitalaria (1-3) y la primera causa de infección entre los pacientes quirúrgicos (38\%) (4); de estas infecciones dos tercios son incisionales y el resto de órgano/espacio. La ISQ causa un impacto importante en términos de mortalidad, morbilidad y costos $(4,5)$; de los pacientes que fallecen por infección postoperatoria, el 77 \% de los casos se originó como ISQ; $93 \%$ de las mismas corresponden a infecciones severas que comprometen órganos o cavidades. De acuerdo a datos del CDC para el año 2002 en los Estados Unidos 8205 de las muertes estuvieron asociadas a ISQ (6). Cada ISQ supone un incremento medio de 7,3 días de estancia postoperatoria y un costo médico directo e indirecto cada año de 1 a 10 billones de dólares(7).

Con el fin de realizar un control de la ISQ, se ha intentado estratificar los principales factores de riesgo descritos en la literatura, tanto prequirúrgicos, intraquirurgicos y del posoperatorio. Inicialmente se considero como parámetro 
la clasificación del grado de contaminación microbiana intrínseca del sitio quirúrgico (8), categorizada en 1980 según un sistema de índice de infección de acuerdo al tipo de herida (9). A pesar que la clasificación de la herida ha mostrado ser un factor predictor independiente de ISQ (10), su utilización en la estratificación de riesgo es limitada, ya que no considera otros factores inherentes al paciente. Existen dos índices NNIS Y SENIC (11) que han mostrado una calificación adecuada del riesgo de infección en la medida que se incrementa de forma paralela tanto el puntaje como la frecuencia de infección del sitio quirúrgico $(12,13)$, sin embargo su desempeño para predecir la aparición de ISQ es motivo de controversia (14). Este desempeño predictivo depende del nivel de complejidad (15), del tipo de cirugía (16) y del índice utilizado $(16,17)$. Aunque se han desarrollado otros índices que toman en cuenta otras variables locales mediante la aplicación de modelos de regresión, los índices NNIS y SENIC siguen siendo el patrón de comparación internacional (18).

En Colombia la validación de los índices SENIC Y NNIS para la predicción de la infección en Bogotá en una Clìnica de segundo nivel de atención mostró que a mayores puntajes se presentó mayor tasa de infección sin embargo muy pocos pacientes con puntajes altos fueron intervenidos (19). No se dispone de un referente significativo respecto a índices de riesgo de acuerdo a cada procedimiento quirúrgico y hay poca información que evalúe otros factores no contemplados en los índices del NNIS y SENIC (19).

Este estudio pretende evaluar el rendimiento de los índices de riesgo del NNIS y SENIC en una población de pacientes atendidos en instituciones privadas de tercer nivel de atención y desarrollar un modelo predictivo que tenga en cuenta las especificidades locales que incrementan el riesgo de infección.

\section{MÉTODOS}

Estudio cohorte de ingreso multicéntrico

Se incluyeron los pacientes sometidos a cirugía que requirieron hospitalización y a cirugía ambulatoria considerada de alto riesgo de infección en tres instituciones generales y dos especializadas en patología cardiovascular de la ciudad de Bogotá. Se excluyeron los pacientes en quienes por algún motivo no se pudiera garantizar el seguimiento en los 30 días post quirúrgicos.

El tamaño de muestra en el que se tomo como factor asociado a ISQ, la cirugía abdominal, mostro una confiabilidad del $95 \%$ y una potencia del $96 \%$; 
y en la construcción del modelo predictivo con el método de regresión logística incondicional de Freeman se tuvo en cuenta para un ajuste adecuado 15 variables predictoras.

Procedimientos

Se realizo un muestreo secuencial o consecutivo de los pacientes.

Posterior a la verificación de los criterios de inclusión y exclusión, antes de que los pacientes fueron a sometidos a cirugía se diligenció el cuestionario de recolección de información por parte de una enfermera integrante del comité de infecciones institucional, especialmente entrenada con este fin. Los datos relacionados con el control postoperatorio se realizaron en los días 15 y 30 por medio de llamada telefónica en la que se buscaron indicadores de infección tales como fiebre, eritema, dolor en la herida o secreción, uso de antibiótico, nombre del antibiótico y días de uso del mismo. Los casos sospechosos de infección durante la hospitalización fueron vistos por el personal del comité de infecciones institucional, quienes confirmaron o descartaron la infección de acuerdo a las definiciones abajo descritas. La información obtenida fue posteriormente enviada al centro de referencia estadística.

Se definió como caso a aquellos sujetos que presentaron los criterios diagnósticos de infección del sitio quirúrgico de acuerdo a los criterios del CDC para la Infección incisional superficial, incisional profunda y de cavidades u órganos (5).

Se incluyeron las siguientes variables: Edad, genero, clasificación de riesgo anestésico (ASA), tipo de herida, tiempo quirúrgico, índice de predicción de ISQ SENIC o NNIS, transfusión sanguínea, desenlace del paciente, comorbilidades, tipo de cirugía y especialidad quirúrgica.

Análisis estadístico: Para el análisis de los datos se utilizó el paquete estadístico SPSS. 15.0. Para determinar las variables asociadas a la aparición de infección se hizo un análisis bivariado. Se estratifico por el sitio de la cirugía. Mediante regresión logística incondicional se ajusto un modelo predictivo de ISQ para la población de estudio. Primero se seleccionaron las variables que mostraron asociación con un valor $<0.2$ en el análisis bivariado, luego se descartó colinealidad entre las variables y se definieron posibles interacciones entre las mismas. Se construyó luego el modelo con las variables seleccionadas y aquellas ya reconocidas como factores de riesgo de infección, que se incluyeron paso a paso mediante el modelo jerárquico. Se evaluó el ajuste con la prueba de bondad de ajuste de Hosmer y Lemeshow y el coeficiente de 
determinación de Nagelkerke y se estableció la probabilidad de predicción que se utilizó posteriormente en la curva operador receptor (ROC) en comparación con el SENIC y NNIS, donde se evaluó el área predictiva de ISQ (Área $>50 \%$ ). También se evaluó la ISQ con las 4 categorías (Superficial, profunda, órgano/espacio y sin ISQ) mediante el modelo de regresión logística multinomial conformándose 3 modelos logit (Supeficial, profunda y órgano/espacio con el grupo sin ISQ) y posteriormente se evaluó su predicción con la curva ROC. Las pruebas estadísticas se evaluaron a un nivel de significancia del $5 \%$ $(\mathrm{p}<0.05)$.

\section{RESULTADOS}

Durante el periodo comprendido entre julio de 2006 y febrero de 2007 fueron evaluados 7022 procedimientos quirúrgicos en 6891 pacientes. Las características demográficas de la población incluida se describen en la Tabla 1. Se presentaron 198 casos de ISQ para una tasa de infección del 2,9 \%. Todos los factores de riesgo incluidos en los índices SENIC y NNIS mostraron una asociación importante excepto la cirugía abdominal (Tablas 2, y 3). El modelo predictivo mostro predicción significativa $(\mathrm{p}<0.001)$, este fue construido involucrando las variables propias del NNIS y SENIC y factores de riesgo locales como la edad, antecedente de diabetes, transfusiones y especialidad quirúrgica, mostró un desempeño operativo de 0,746 (IC95 \% 0,709-0,783), mostrando ajuste ( $\mathrm{p}=0,881$, Test de Hosmer y Lemeshow) y un coeficiente de determinación de Nagelkerke del 10,9 \%. A continuación se presenta:

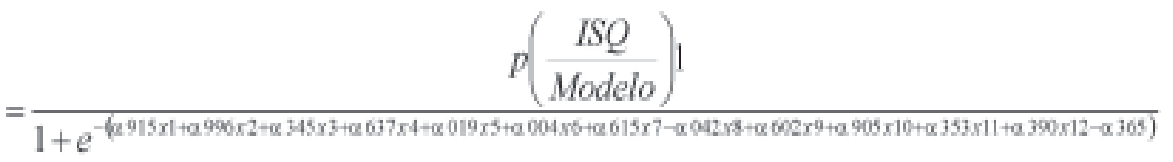

X1: Herida contaminada o sucia; X2: Cirugía abdominal; X3: Diabetes Mellitus; X4: Transfusiones; X5: Edad; X6: Duración de la cirugía; X7: Cirugía general; X8: Cirugía pediátrica; X9: Ortopedia; X10: Cirugía vascular y cardiovascular; X11: Cirugía plástica-Otorrino; X12: ASA >= 3

Las características de los índices de riesgo para la predicción de infección mostraron un área bajo la curva de 0,682 (IC95 \% 0,627-0,710) para NNIS y 0,668 (IC95 \% 0,641-0,722) para SENIC, con los siguientes modelos: 


$$
\begin{gathered}
p\left(\frac{I S Q}{S E N I C}\right)=\frac{1}{1+e^{-(0,429.51+1.705 \times 2+2.704 \times 3+2.798 \times 4-4,303)}} \\
p\left(\frac{I S Q}{N N I S}\right)=\frac{1}{1+e^{-(0,808 \times 1+1.697 \times 2+2.953 \times 3-4.206)}}
\end{gathered}
$$

En los tres modelos logit se mostró predicción significativa ( $<<0.001)$, en el logit de ISQ superficial una área 0,70 (IC95 \% 0,659-0,741), en ISQ profunda de 0,712 (IC95 \% 0,673-0,751) y en ISQ órgano espacio de 0,719 (IC95 \% 0,683-0,755). El área de predicción de los diferentes modelos se presenta en la Figura 1.

Tabla 1. Factores asociados a infección quirúrgica en instituciones

\begin{tabular}{|c|c|c|c|}
\hline \multirow{2}{*}{ Variable } & ISQ & No ISQ & \multirow[t]{2}{*}{ valor $p$} \\
\hline & No. (\%) & No, (\%) & \\
\hline 0 a 11.9 años & $3(1,3)$ & $236(98,7)$ & $<0.001$ \\
\hline 12 a 19.9 aกิంs & $2(0.9)$ & $221(99.1)$ & \\
\hline 20 a 44.9 años & $41(1,6)$ & $2600(98.4)$ & \\
\hline 45 а 69.9 คน̆० & $100(3.7)$ & 2581 (96.3) & \\
\hline 70 o mas años & $53(4,8)$ & $1054(95.2)$ & \\
\hline \multicolumn{4}{|l|}{ Genero } \\
\hline Masoulino & $91(3,9)$ & $2229(98,1)$ & $<0.001$ \\
\hline Femenino & $108(2.4)$ & $4463(97.6)$ & \\
\hline \multicolumn{4}{|l|}{ Especialidad } \\
\hline Cirugia general & $90(4,2)$ & $2071(85.8)$ & $<0.001$ \\
\hline Cirugia pediatrica & $1(1,0)$ & $102(99)$ & \\
\hline Ortopedia & $35(2,4)$ & $1454(97,6)$ & \\
\hline \multicolumn{3}{|l|}{ Cirugia vasculary } & \\
\hline Cirugia plástica - Otorrino & $6(1,5)$ & $400(98,5)$ & \\
\hline Ginecobstetricia & $33(1,4)$ & 2303 (90,6) & \\
\hline \multicolumn{4}{|l|}{ Tipo de herida } \\
\hline Limpia & $67(2,8)$ & $2308(97,2)$ & $<0.001$ \\
\hline Limpia Contaminada & $99(2,4)$ & $4061(97,6)$ & \\
\hline Contaminada & $34(7,2)$ & $437(92,8)$ & \\
\hline Sucia & $0(0)$ & $16(100)$ & \\
\hline Duración cirugia (minutos) & $146 \pm 93$ & $99 \pm 75$ & $<0.001$ \\
\hline \multicolumn{4}{|l|}{ Calificacion ASA } \\
\hline$<3$ & $120(2,1)$ & $5681(97,9)$ & $<0,001$ \\
\hline$>=3$ & $80(6,6)$ & $1141(98,4)$ & \\
\hline Cirugia abdominal & $122(3,1)$ & $96,9(3841)$ & 0.106 \\
\hline Patologia asociada & $127(4.7)$ & $98,3(4258)$ & $<0.001$ \\
\hline
\end{tabular}
de tercer nivel de atención en Bogotá, Colombia 2006 
Tabla 2. Factores de riesgo del índice SENIC asociados a infección quirúrgica en instituciones de tercer nivel de atención en Bogotá Colombia

\begin{tabular}{lccccc}
\multicolumn{5}{c}{2006} \\
\hline Factor & RR & $\begin{array}{l}\text { Limile } \\
\text { inferior }\end{array}$ & $\begin{array}{c}\text { Limite } \\
\text { superior }\end{array}$ & $\begin{array}{c}\text { Exp. vs. No exp } \\
\%\end{array}$ & Significancia \\
\hline $\begin{array}{l}\text { Mas de 3 } \\
\text { antecedentes } \\
\text { patologicos }\end{array}$ & 3,7 & 2,5 & 5,5 & 9,6 vs. 2,6 & $<0,001$ \\
$\begin{array}{l}\text { Crugia }>=2 \\
\text { horas }\end{array}$ & 2,8 & 2,1 & 3,6 & 5,1 v8. 1,8 & $<0.001$ \\
$\begin{array}{l}\text { Crugia } \\
\text { contaminada } \\
\text { o sucia }\end{array}$ & 2,7 & 1,9 & 4,2 & 7,0 vs. 2,5 & $<0.001$ \\
$\begin{array}{l}\text { Crugia } \\
\text { abdominal }\end{array}$ & 1,2 & 0,9 & 1,6 & 3,1 vs. 2,5 & 0.106 \\
\hline
\end{tabular}

Tabla 3. Factores de riesgo del índice NISS asociados a infección quirúrgica en instituciones de tercer nivel de atención en Bogotá Colombia 2006

\begin{tabular}{|c|c|c|c|c|c|}
\hline Factor & RR & Limite inferior & $\begin{array}{l}\text { Limite } \\
\text { superior }\end{array}$ & Exp, vs. No exp \% & Significancia \\
\hline $\begin{array}{l}\text { Cirugia }>=2 \\
\text { horas }\end{array}$ & 2.8 & 2,1 & 3,6 & 5.1 vs. 18 & $<0.001$ \\
\hline ASA $3,4,5$ & 3.2 & 2,4 & 4,1 & 6.6 v8. 2.1 & $<0.001$ \\
\hline $\begin{array}{l}\text { Herida } \\
\text { comtaminada } \\
\text { o sùcia }\end{array}$ & 2,7 & 1,9 & 4.2 & 7.0 v8. 2.5 & $<0.001$ \\
\hline
\end{tabular}

Figura 1. Curva COR. Desempeño operativo de los Índices SENIC NISS y local en pacientes operados en instituciones de tercer nivel de atención en Bogotá, Colombia 2006

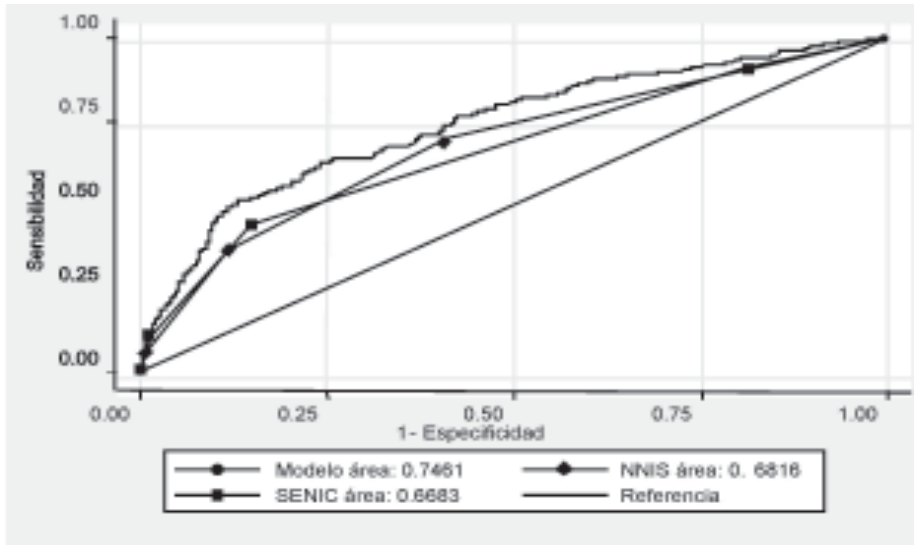

\section{DISCUSIÓN}

La vigilancia de ISQ ha demostrado ser un factor primordial en la reducción de la tasa de infección y para ello a través del tiempo se han usado diferentes comparadores siendo el más popular en la actualidad el índice de riesgo NNIS. 
Sin embargo, este índice tiene debilidades importantes como el hecho de incluir variables como el ASA que clasifica el riesgo anestésico y no el riesgo de infección, darle el mismo peso a todas las variables y no tener en cuenta diferencias de factores de riesgo acorde a los diferentes procedimientos, entre otros, por lo que se ha recomendado optimizar su aplicación buscando modelos acordes a situaciones locales, tipos de procedimientos y factores de riesgo intrínsecos a cada tipo de intervención (20).

En el presente estudio en el que realizamos la validación de los índices SENIC y NNIS y proponemos un modelo de predicción para ISQ aplicable a nuestros pacientes, encontramos que las variables que los conforman de forma individual mostraron ser un factor de riesgo para ISQ, a excepción de la herida abdominal. El factor de riesgo de los dos índices que presentó mayor importancia fue la presencia de más de dos antecedentes patológicos. Entre los componentes del índice de riesgo NNIS, el riesgo de cada uno de los factores observado en este trabajo es similar a lo descrito en otros trabajos similares realizados en Colombia, España y Brasil $(25,27)$. Así mismo, se observo un incremento progresivo en riesgo de ISQ en los dos índices a medida que se aumentan la positividad de los criterios que los conforman, hallazgo esperable y documentado en la literatura.

En un estudio previo realizado en Colombia la validación de los índices SENIC y NNIS para la predicción de la infección en Bogotá en una Clínica de segundo nivel de atención, mostró que a mayores puntajes se presentó mayor tasa de infección, sin embargo muy pocos pacientes con puntajes altos fueron intervenidos, adicionalmente el único factor que resulto crítico fue el tiempo de infección, los otros factores no fueron relevantes posiblemente por las características de la Institución (19). No se dispone de un referente significativo respecto a índices de riesgo de acuerdo a cada procedimiento quirúrgico y hay poca información que evalúe otros factores no contemplados en los índices del NNIS y SENIC.

Recientemente, Neumayer L y col. proponen un nuevo índice predictivo con 14 variables que de acuerdo a sus resultados es mucho más preciso que el NNIS (0,7 Vs 0,62 respectivamente). Por otra parte, Geubbels y col. validan un nuevo modelo predictivo adaptado para 5 procedimientos, demostrando mayor precisión de cada uno de ellos con respecto al NNIS ( 0,75 vs 0,62 para reconstrucción de la aorta; 0,78 Vs 0,58 para bypass femoropopliteo; 0,69 Vs 0,62 para colectomia; 0,64 Vs 0,56 para artoplastia con prótesis de cadera y 0,70 Vs 0, 54 para cesárea). Este modelo al igual que el modelo propuesto por 
Neumayer tiene la desventaja en nuestra opinión de ser complejo y difícil de aplicar en programas de vigilancia epidemiológica con recursos humanos y técnicos limitados, en los que la recolección y análisis de esta información de forma rutinaria los hacen poco prácticos.

En Suramérica existen pocos estudios que caractericen la situación actual respecto a ISQ y que establezcan tasas ajustadas a riesgo (22-26). Arias y col, describen las tasas de infección de acuerdo a la clasificación del NNIS y el SENIC en un centro universitario de tercer nivel durante 10 años. En otro estudio Morales y colaboradores establecieron los tiempos quirúrgicos en una institución de tercer nivel y evaluaron el riesgo NNIS para los procedimientos de mayor complejidad encontrando algunos procedimientos con valores superiores a los descritos en la literatura. Así mismo, Hernández K y col. describieron la incidencia de ISQ en un hospital peruano y algunos factores de riesgo asociados, encontrando también una mayor incidencia de lo descrito en el NNIS. Un estudio en Brasil, demostró una disminución en la tasa de ISQ durante 10 años de seguimiento basado en un programa de supervisión y además encontraron asociación directa entre la tasa de ISQ y las 3 variables incluidas en el NNIS (25). La validación de los índices SENIC y NNIS para la predicción de ISQ en una Clínica de segunda nivel de atención en Bogotá, mostró que a mayores puntajes se presento mayor tasa de infección, sin embargo se incluyeron muy pocos pacientes con puntajes altos. El único factor que resulto crítico fue el tiempo de infección, los otros factores no fueron relevantes posiblemente por las características de la Institución.

En nuestro estudio las variables que conforman los índices SENIC y NNIS de forma individual mostraron ser un factor de riesgo para ISQ, a excepción de la herida abdominal. El factor de riesgo de los dos índices que presento mayor importancia fue la presencia de más de dos antecedentes patológicos. Entre los componentes del índice de riesgo NNIS, el riesgo de cada uno de los factores observado en este trabajo es similar a lo descrito en otros trabajos similares realizados en Colombia, España y Brasil (25,27). Así mismo, se observo un incremento progresivo en riesgo de ISQ en los dos índices a medida que se aumentan la positividad de los criterios que los conforman, hallazgo esperable y documentado en la literatura.

En general el área bajo la curva ROC se puede considerar el mejor estimador del rendimiento global de una prueba diagnóstica o predictora para estimar la proporción de eventos clasificados correctamente. En nuestro estudio se encontró que la capacidad predictiva de los índices de riesgo existentes (NNIS 
y SENIC) es moderada sin encontrar diferencias significativas entre ellos, contrario a lo descrito por Delgado_Rodriguez y col, quienes al evaluar diferentes desenlaces (ISQ, otras infecciones nosocomiales, muerte, etc) evidenciaron una mayor habilidad predictora del índice NNIS. Al incluir otras variables como la edad, la presencia de diabetes, administración de transfusiones y especialidad quirúrgica a las variables incluidas en el SENIC y NNIS, se obtuvo un mejor desempeño predictivo de nuestro modelo e incluso superior a los encontrados por Neumayer y Geubbels y a la validación del índice NNIS en 13 procedimientos quirúrgicos realizados en centros hospitalarios australianos, en el que encontraron una sensibilidad que variaba entre $0,47-0,69$ y una especificidad entre 0,51 y 0,93 (28).

Finalmente el modelo propuesto por nosotros tiene la ventaja de ser más sencillo que los otros descritos en la literatura con la inclusión sólo de 4 variables fáciles de obtener en la historia clínica por lo que creemos que puede ser rápidamente implementado en los servicios quirúrgicos y de vigilancia epidemiológica tanto para evaluar el riesgo de ISQ de forma individual como para comparar tasas entre instituciones. La posibilidad de poder discriminar con mayor precisión el riesgo individual de ISQ permitirá establecer medidas preventivas para aquellos pacientes con un alto riesgo y de esta manera lograr un impacto en la tasa de ISQ global.

Si se aplica la ecuación que a continuación proponemos en un paciente determinado se podría fácilmente obtener su riesgo.

Riesgo infección=Edad x 0,019 + Herida contaminada x 0,815 + Cirugía abdominal x 0,996 + DM x 0,345 + Transfusiones x 0,637 + ASA x 0,380 + duración de La cirugía x 0,004+ Cx general x 0,615 +cirugía pediátrica x$0,042+$ Ortopedia x $0,602+$ Cx vascular y cardiovascular x $0,905+$ Cx plástica x $0,353+$ otorrino x 6,365).

En el caso de un paciente de 65 años con ASA >3, con antecedentes de DM e HTA a quien que se le practicó una hemicolectomia izquierda por un Ca de Recto, con una duración de cirugía de 1 hora y 30 minutos y quien no requirió transfusiones y presento herida contaminada, tiene un riesgo de presentar ISQ del 18,9 \% aplicando la fórmula propuesta de la siguiente manera:

Riesgo infección $=65$ x 0,019 + Herida contaminada 1 x 0,815 + Cirugía abdominal $1 \times 0,996+$ DM x 0,345 + Transfusiones $0 \times 0,637+$ ASA $1 \times 0,380+$ duración de la cirugía 130 x 0,004+Cirugía general 1 x 0,615 - 6,365. 
No obstante se debe resaltar que para la interpretación de los resultados de este estudio se debe tener en cuenta que las instituciones seleccionadas no reflejan la situación de la ciudad de Bogotá, dado que no involucran instituciones públicas, las cuales atienden población con características socioeconómicas diferentes que podrían incidir tanto en el riesgo de ISQ como en la frecuencia de procedimientos realizados; adicionalmente se debe tener en cuenta que no se realizó estandarización de la variable del tiempo quirúrgico del NNIS basado en el percentil $75 \%$ como es determinado en la actualidad.

Por otra parte, al comparar los resultados de este estudio con otros descritos en la literatura se debe tener en cuenta que hay diferencias en la población estudiada y la metodología seleccionada por lo que más que comparar los resultados de este estudio con algunos publicados sugerimos evaluar la aplicación de este modelo en poblaciones similares, e intentar validar este modelo propuesto en otros escenarios como los descritos anteriormente *

Agradecimientos. Al Instituto Colombiano de Ciencia y Tecnología COLCIENCIAS que financió este proyecto mediante el Grant identificado con el código: 6123-0416523. Grupo Vigilancia Epidemiológica de Infecciones Quirúrgicas: Clínica Reina Sofía: Nancy Barrera Niño; Clínica Palermo: Patricia Hernández; Clínica Fundación Abood Shaio: Angela Novoa, Julia Ortiz; Clínica Nueva: Betty Mojica; Clínica Fundación CardioInfantil: Zenaida Montañez y Andrea Galindo.

\section{REFERENCIAS}

1. Martone WJ, Jarvis WR, Culver DH, Haley RW. Incidence and nature of endemic and epidemic nosocomial infections. In: Bennett JV, Brachman PS, eds. Hospital infections. Third ed. Boston: Little, Brown and Co; 1992. pp. 577-596.

2. National Nosocomial Infections Surveillance (NNIS) system report data summary from January 1992-June 2001, issued August 2001. Am J Infect Control 2001;29:404-421.

3. Emori TG, Gaynes RP. An overview of nosocomial infections, including the role of the microbiology laboratory. Clin Microbiol Rev, 1993; 6(4): 428-42.

4. Gaynes R. Feeding Back Surveillance Data To Prevent Hospital-Acquired Infections. Emerging Infectious Diseases 2001;7(2):295-300.

5. Mangram AJ, Horan TC, Pearson ML, Silver RC, Jarvis WR. The Hospital Infection Control Practices Advisory Committee. Guideline for the prevention of surgical site infection, 1999. Infect Control Hosp Epidemiol 1999; 20: 247-280.

6. Klevens RM, Edwards JR, Richards CL Jr, Horan TC, Gaynes RP, Pollock DA, Cardo DM. Estimating health care-associated infections and deaths in U.S. hospitals, 2002. Public Health Rep. 2007;122(2):160-6.

7. Holtz TH, Wenzel RP. Postdischarge surveillance for nosocomial wound infection: a brief review and commentary. Am J Infect Control 1992;20:206-13.

8. National Academy of Sciences, National Research Council, Division of Medical Sciences, Ad Hoc Committee on Trauma. Postoperative wound infections: the influence of ultraviolet irradiation on the operating room and of various other factors. Ann Surg 1964;2:1. 
9. Cruse PJ, Foord R. The epidemiology of wound infection: a 10-year prospective study of 62,939 wounds. Surg Clin North Am 1980;60:27-40.

10. Garibaldi RA, Cushing D, Lerer T. Risk factors for postoperative infection. Am J Med 1991;91(Suppl 3B):158S-163S.

11. Haley RW, Culver DH, Morgan WM, White JW, Emori TG, Hooton TM. Identifying patients at high risk of surgical wound infection. A simple multivariate index of patient susceptibility and wound contamination. Am J Epidemiol. 1985;121:206-15.

12. Emori TG, Culver DH, Horan TC, Jarvis WR, White JW, Olson DR, et al. National Nosocomial Infections Surveillance (NNIS) System: Description of surveillance methodology. Am J Infect Control 1991;19:19-35.

13. CDC NNIS System. National Nosocomial Infections Surveillance (NNIS) system report, data summary from January 1992 to June 2004, issued October 2004. Am J Infect Control 2004;32:470-85.

14. Ercole FF, Starling CE, Chianca TC, Carneiro M. Applicability of the national nosocomial infections surveillance system risk index for the prediction of surgical site infections: a review. Braz J Infect Dis. 2007;11:134-41.

15. Fajardo RHA, Quemba GJ, Eslava Schmalbach J. Prediction scales and infection on surgical sites in 15625 surgeries. 2001-2003. Rev Salud Publica (Bogota). 2005 Jan-Apr;7(1):8998.

16. Neumayer L, Hosokawa P, El-Tamer M, Henderson WG, Khuri, SF. Multivariable Predictors of Postoperative Surgical Site Infection after General and Vascular Surgery: Results from the Patient Safety in Surgery Study. J Am Coll Surg 2007;204:1178-1187.

17. Delgado-Rodriguez M, Palma S, Gomez-Ortega A, Martinez-Gallego G, Medina-Cuadros M. Indices of surgical site infection risk and prediction of other adverse outcomes during hospitalization. Infect Control Hosp Epidemiol. 2006;27(8):825-8.

18. Haas JP. Measurement of infection control department performance: state of the science. Am J Infect Control. 2006;34:543-9.

19. Eslava J, Saavedra C, Cardona J, Quemba J, Porras N, Velásquez O. et al. Vigilancia Epidemiológica de infecciones intrahospitalarias en la Clínica Carlos Lleras Restrepo del Seguro Social (CCLIR) en Bogotá. Boletín Epidemiologico Distrital. 2001;6:1-24.

20. Gaynes RP. Surgical-Site Infections and the NNIS SSI Risk Index: Room for Improvement. Infect Control Hosp Epidemiol 2000;21(3):184-85.

21. Geubbels E, Grobbee DE, Vandenbroucke-Grauls CE, Wille JC, Boer A.Improved RiskAdjustment for Comparison of Surgical Site Infection Rates. Infect Control Hosp Epidemiol. 2006; 27:1330-1339.

22. Morales S, López C, Moreno L, Munevar M, Linares C, Alvarez C. Infección del sitio operatorio en un hospital de tercer nivel. Universitas Médica 2005;46(2):42-47.

23. Arias CA, Quintero G, Vanegas BE, Rico CL, Patiño JF. Surveillance of surgical site infections: decade of experience at a Colombian tertiary care center. World J Surg.2003 May;27(5):529-33.

24. Del Río JA. Buriticá OC. Evaluación de un sistema de vigilancia epidemiológica y riesgos de infección intrahospitalaria en pacientes quirúrgicos. Colombia Med 2000; 31: 71-6.

25. Medeiros AC, Aires-Neto T, Azevedo GD, Vilar MJ, Pinheiro LA, Brandão-Neto J. Surgical site infection in a university hospital in northeast Brazil. Braz J Infect Dis. 2005;9(4):310-4.

26. Hernandez K, Ramos E, Seas C, Henostroza G, Gotuzzo E. Incidence of and risk factors for surgical-site infections in a Peruvian hospital. Infect Control Hosp Epidemiol. 2005 May;26(5):473-7.

27. Íñigo J, Begoña B, Begoña O, Herrera J, TarifaA, Pérez F, Miranda C, Lera JM. Infección de sitio quirúrgico en un servicio de cirugía general. Análisis de cinco años y valoración del índice National Nosocomial Infection Surveillance (NNIS). Cir Esp. 2006;79(4):224-30.

28. Clements AC, Tong EN, Morton AP, Whitby M. Risk stratification for surgical site infections in Australia: evaluation of the US National Nosocomial Infection Surveillance risk index. J Hosp Infect. 2007 Jun;66(2):148. 